\title{
LOCAL HARNACK ESTIMATE FOR YAMABE FLOW ON LOCALLY CONFORMALLY FLAT MANIFOLDS*
}

\author{
SHOUWEN FANG ${ }^{\dagger}$
}

\begin{abstract}
In this paper, we first prove the local derivative estimate of curvature under Yamabe flow, and then by using it obtain the local Harnack estimate of Yamabe flow on locally conformally flat manifolds, under the condition $-m(t) g_{a b} \leq R_{a b} \leq M g_{a b}$, where $0 \leq m(t) \leq M$ and $m^{\prime}(t) \geq$ $(4 n+1) m(t) M$, on $t \in\left[0, r^{2}\right]$. As a corollary, we get a sharp derivative estimate of scalar curvature in some directions.
\end{abstract}

Key words. Yamabe flow, Local Harnack estimate.

AMS subject classifications. $53 \mathrm{C} 21,53 \mathrm{C} 44$

1. Introduction. The Harnack estimate of geometry flow is also called Li-YauHamilton inequality. R.Hamilton made important work on the Harnack estimate of Ricci flow and mean curvature flow. Recently he has found the local Harnack estimate of Ricci flow under the curvature condition $-\frac{m(t)}{2}\left(g_{a c} g_{b d}-g_{a d} g_{b c}\right) \leq R_{a b c d} \leq$ $\frac{M}{2}\left(g_{a c} g_{b d}-g_{a d} g_{b c}\right)$, where $0 \leq m(t) \leq M$. It can be used to prove the second step of the proof of the Theorem7.1.1 in [1]. That was his report on the fourth ICCM in December 2007. Jie Wang got the local Harnack estimate of mean curvature flow by using the same method in [4]. The Harnack inequality of Yamabe flow was first proved by Chow on compact locally conformally flat manifolds with positive Ricci curvature in [2]. HuiLing Gu obtained the same inequality for complete locally conformally flat manifolds with nonnegative Ricci curvature in [3]. Does the local Harnack estimate also hold for the Yamabe flow ? We give an affirmative answer in this paper in the class of locally conformally flat manifolds.

Let $\left(M^{n}, g_{0}\right)$ be a smooth complete locally conformally flat $n$-dimensional manifold. The Yamabe flow is given by

$$
\left\{\begin{array}{l}
\frac{\partial}{\partial t} g(x, t)=-R(x, t) g(x, t) \\
g(x, 0)=g_{0}(x)
\end{array}\right.
$$

for $x \in M^{n}, t \geq 0$, and where $R$ is the scalar curvature of $g$.

Let $0 \leq r \leq \theta / \sqrt{M}$, where constant $\theta$ depending only on the dimension $n$ will be obtained in Theorem 2.1. Let $O \in M^{n}, B_{r}(O, t)$ is a geodesic ball centered at $O$, with radius $r$ at time $t$. Set $d=d_{t}(x, O)$ is the geodesic distance function from $O$ to $x$ w.r.t. $g_{i j}(t)$.

Through out the paper, we denote the curvature condition

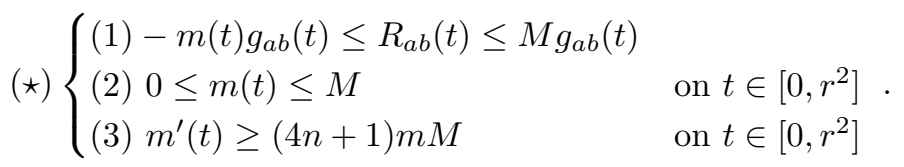

Our main result is the following theorem:

\footnotetext{
*Received May 20, 2008; accepted for publication July 10, 2008.

${ }^{\dagger}$ Center of Mathematics and Science, Zhejiang University, Hangzhou, Zhejiang, 310027, P. R. China (shwfang@163.com).
} 
THEOREM 1.1. Suppose $\left(M^{n}, g(x, t)\right)$ is a smooth complete locally conformally flat $n$-dimensional manifold for $t \in\left[0, r^{2}\right]$, the curvature satisfies the condition $(\star)$ on $B_{r}(O, t) \times\left[0, r^{2}\right]$, then at $\forall(x, t) \in B_{\frac{r}{2}}(O, t) \times\left[0, r^{2}\right], \forall V \in T_{x} M^{n}$, we can find some constant $B>0$, depending only on $n$, such that the following local Harnack estimate holds,

$$
\begin{aligned}
\partial_{t} R & +n m(t) R+\nabla R(V)+\frac{1}{2(n-1)}\left(R_{a b}+m(t) g_{a b}\right) V_{a} V_{b} \\
& +B M\left(M+\frac{r^{2}}{\left(r^{2}-4 d^{2}\right)^{2}}+\frac{1}{t}\right) \geq 0 .
\end{aligned}
$$

As a corollary we get a sharp gradient estimate of scalar curvature.

Corollary 1.1. Under the same conditions as Theorem 1.1, at point $\left(O, r^{2}\right)$, we have

(1) $|\nabla R(V)|^{2} \leq C_{1} M^{2}\left(R_{a b}+m(t) g_{a b}\right) V_{a} V_{b}, \quad M \geq 1$

(2) $|\nabla R(V)|^{2} \leq C_{2}\left(R_{a b}+m(t) g_{a b}\right) V_{a} V_{b}, \quad 0<M<1$

(3) $\nabla R(V)=0$, if $\left[\partial_{t} R+n m(t) R+B M\left(1+\frac{1}{r^{2}}+\frac{1}{t}\right)\right]\left(O, r^{2}\right)=0$ or $\left(R_{a b}+\right.$ $\left.m(t) g_{a b}\right) V_{a} V_{b}\left(O, r^{2}\right)=0$

where $C_{1}$ and $C_{2}$ depend only on $n$.

The paper is organized as follows. In Section 2, we obtain the local derivative estimate of curvature under the Yamabe flow. In Section 3 and 4, we compute the evolution equation of the Harnack quantity, find a good positive term in the equation, and then estimate another extra term. The proofs of Theorem 1.1 and its corollary will be given in Section 5 .

2. Local derivative estimate of curvature. In this section we will obtain the local derivative estimate of curvature as Ricci flow. Let $M^{n}$ be a smooth complete $n$ dimensional manifold. Under the Yamabe flow (1.1), we give the evolution equations of curvature as follows.

LEMMA 2.1. If $\left(M^{n}, g\right)$ is locally conformally flat manifold, then

(1) $\partial_{t} R_{i j k l}=(n-1) \triangle R_{i j k l}-R R_{i j k l}+\frac{1}{(n-2)^{2}}\left(B_{i k} g_{j l}+B_{j l} g_{i k}-B_{i l} g_{j k}-B_{j k} g_{i l}\right)$,

(2) $\partial_{t} R_{i j}=(n-1) \triangle R_{i j}+\frac{1}{n-2} B_{i j}$,

(3) $\partial_{t} R=(n-1) \triangle R+R^{2}$, where $B_{i j}=(n-1)|R i c|^{2} g_{i j}+n R R_{i j}-n(n-1) R_{i j}^{2}-R^{2} g_{i j}$.

The proof of Lemma 2.1 can be found in [3] and [2]. The third equation also holds without the locally conformally flat condition. Let us denote the Ricci curvature or curvature tensor by $R m$ and denote by $A * B$ any tensor product of two tensor $A$ and $B$. We can get the following result from Lemma 2.1.

LEMMA 2.2. If $\left(M^{n}, g\right)$ is locally conformally flat manifold, then for $\forall k \in \mathbb{Z}$, $k \geq 0$ we have

$$
\begin{aligned}
& \text { (1). } \quad \frac{\partial}{\partial t}\left(\nabla^{k} R m\right)=(n-1) \triangle\left(\nabla^{k} R m\right)+\sum_{i+j=k} \nabla^{i} R m * \nabla^{j} R m \\
& \text { (2). } \frac{\partial}{\partial t}\left(\left|\nabla^{k} R m\right|^{2}\right)=(n-1) \triangle\left|\nabla^{k} R m\right|^{2}-2(n-1)\left|\nabla^{k+1} R m\right|^{2} \\
& +\sum_{i+j=k} \nabla^{k} R m * \nabla^{i} R m * \nabla^{j} R m .
\end{aligned}
$$


The proof is just direct calculation by induction. By using a cutoff argument as Ricci flow, we will get a local derivative estimate of curvature.

TheOREM 2.1. There exist positive constant $\theta, C_{k}, k=1,2, \cdots$, depending only on the dimension with the following property. Suppose that the curvature of a complete locally conformally flat solution to the Yamabe flow is bounded

$$
|R m|<M \quad \text { on } \quad U \times[0, \theta / M]
$$

where $U$ is an open set of the manifold. Assume that the closed ball $B_{r}(p, 0)$ is contained in $U$ and the time $t \leq \theta / M$. Then we can estimate the covariant derivative of the curvature at $(p, t)$ by

$$
|\nabla R m(p, t)|^{2} \leq C_{1} M^{2}\left(\frac{1}{r^{2}}+\frac{1}{t}+M\right),
$$

and the $k^{\text {th }}$ covariant derivative of the curvature at $(p, t)$ by

$$
\left|\nabla^{k} R m(p, t)\right|^{2} \leq C_{k} M^{2}\left(\frac{1}{r^{2 k}}+\frac{1}{t^{k}}+M^{k}\right)
$$

Proof. Without loss of generality, we may assume the exponential map at $\mathrm{p}$ at time $\mathrm{t}=0$ is injective on the ball of radius $r$. From the Lemma 2.2, we get the following inequalities

$$
\begin{aligned}
\frac{\partial}{\partial t}\left(|R m|^{2}\right) & \leq(n-1) \triangle\left(|R m|^{2}\right)-2(n-1)|\nabla R m|^{2}+C|R m|^{3} \\
\frac{\partial}{\partial t}\left(|\nabla R m|^{2}\right) & \leq(n-1) \triangle|\nabla R m|^{2}-2(n-1)\left|\nabla^{2} R m\right|^{2}+C|R m||\nabla R m|^{2}
\end{aligned}
$$

for some constant $\mathrm{C}$ depending only on the dimension $n$.

In Ricci flow we have the similar inequalities with the above ones. Thus the rest of argument is similar with Ricci flow in [1]. We need only to make a little corresponding change and the theorem follows.

3. Good extra term. In this section, we will derive the evolution equation of the basic Harnack expression under the Yamabe flow (1.1), then find a good positive term in the evolution equation.

Let $\left\{E_{a}\right\},(a=1,2, \ldots, n)$ be an orthonormal frame locally, where $E_{a}=E_{a}^{i} \frac{\partial}{\partial x^{i}}$ is tangent to $M$. To keep the vectors orthonormal and tangent to $M$ under the Yamabe flow, we let

$$
\frac{\partial}{\partial t} E_{a}=\frac{1}{2} R E_{a}
$$

We can write the components of tensors in terms of the moving frame, for example $R_{a b}=R\left(E_{a}, E_{b}\right)$ or in local coordinates, $R_{a b}=R_{i j} E_{a}^{i} E_{b}^{j}$. We always denote indices $a, b, c \ldots$ under orthonormal frame, and $i, j, k \ldots$ under local coordinates.

For convenience, now we take covariant derivatives under the moving orthonormal frame, for example $\nabla_{a} V_{b}=E_{a}^{i} E_{b}^{j} \nabla_{i} V_{j}$. Then the Laplacian is $\Delta=\sum_{a} \nabla_{a} \nabla_{a}$. We define the operator $\square=\partial_{t}-(n-1) \triangle$. 
DeFinition 3.1. We let

$$
\begin{aligned}
& X_{a}=\frac{1}{2} \nabla_{a} R+\frac{1}{2(n-1)} R_{a b} V_{b}, \quad Y_{a b}=\nabla_{a} V_{b}-R g_{a b}, \\
& W_{a b}=(n-1) \nabla_{a} \nabla_{b} R+\nabla_{a} R_{b c} V_{c}+R R_{a b}, \\
& Z=\partial_{t} R+V_{a} \nabla_{a} R+\frac{1}{2(n-1)} R_{a b} V_{a} V_{b}, \quad W=\partial_{t} R+\frac{1}{2} \nabla_{a} R V_{a}, \\
& U_{a}=\square V_{a}-\frac{(n-1)(n-2)}{2} \nabla_{a} R-\frac{n}{2} R_{a b} V_{b}+R V_{a} .
\end{aligned}
$$

Now we can take the computation directly.

THEOREM 3.1. For any locally conformally flat solution to the Yamabe flow and any vector field $V$, we have:

$$
\square Z=R Z+2 X_{a} U_{a}+A_{a b} V_{a} V_{b}-R_{a b} Y_{c a} Y_{c b}-2 W_{a b} Y_{a b},
$$

where $A_{a b}=\frac{1}{2(n-1)(n-2)} B_{a b}+\frac{1}{2(n-1)}\left(n R_{a b}^{2}-R R_{a b}\right)$.

Proof. The computation is not difficult and we can refer to [2] for more details,

$$
\begin{aligned}
\square Z= & 3 R Z-R^{3}+\frac{1}{2(n-1)(n-2)} B_{a b} V_{a} V_{b}-\frac{1}{2(n-1)} R R_{a b} V_{a} V_{b} \\
& -\frac{(n-1)(n-2)}{2}|\nabla R|^{2}-(n-1) R_{a b} \nabla_{a} R V_{b}+\nabla_{a} R \square V_{a}+\frac{R_{a b} V_{b} \square V_{a}}{n-1} \\
& -R_{a b} \nabla_{c} V_{a} \nabla_{c} V_{b}-2 \nabla_{c} R_{a b} \nabla_{c} V_{a} V_{b}-2(n-1) \nabla_{a} \nabla_{b} R \nabla_{a} V_{b} \\
= & 3 R Z-R_{a b} Y_{c a} Y_{c b}-2\left[R R_{a b}+(n-1) \nabla_{a} \nabla_{b} R+\nabla_{a} R_{b c} V_{c}\right] \nabla_{a} V_{b} \\
& +\nabla_{a} R\left(\square V_{a}-\frac{(n-1)(n-2)}{2} \nabla_{a} R-\frac{n}{2} R_{a b} V_{b}\right)+\frac{1}{n-1} R_{a b} V_{b}\left(\square V_{a}\right. \\
& \left.-\frac{(n-1)(n-2)}{2} \nabla_{a} R\right)+A_{a b} V_{a} V_{b}-\frac{n}{2(n-1)} R_{a b}^{2} V_{a} V_{b} \\
= & 3 R Z-R_{a b} Y_{c a} Y_{c b}-2 W_{a b} Y_{a b}-2 R W+2 X_{a} U_{a}-2 R X_{a} V_{a}+A_{a b} V_{a} V_{b} \\
= & R Z+2 X_{a} U_{a}+A_{a b} V_{a} V_{b}-R_{a b} Y_{c a} Y_{c b}-2 W_{a b} Y_{a b} .
\end{aligned}
$$

We now replace the $Y_{a b}$ with $\widetilde{Y_{a b}}=Y_{a b}+\widetilde{E_{a b}}$. Then

$$
\begin{aligned}
\square Z= & R Z+2 X_{a} U_{a}+A_{a b} V_{a} V_{b}-R_{a b} \widetilde{Y_{c a}} \widetilde{Y_{c b}}-2 W_{a b} \widetilde{Y_{a b}}+2 R_{a b} \widetilde{E_{c a}} \widetilde{Y_{c b}} \\
& +2 W_{a b} \widetilde{E_{a b}}-R_{a b} \widetilde{E_{c a}} \widetilde{E_{c b}} .
\end{aligned}
$$

We set $\widetilde{E_{a b}}=R_{b c}^{-1} W_{a c}$. So

$$
-2 W_{a b} \widetilde{Y_{a b}}+2 R_{a b} \widetilde{E_{c a}} \widetilde{Y_{c b}}=-2 W_{a b} \widetilde{Y_{a b}}+2 R_{a b} R_{a d}^{-1} W_{c d} \widetilde{Y_{c b}}=0
$$

and

$$
\begin{aligned}
2 W_{a b} \widetilde{E_{a b}}-R_{a b} \widetilde{E_{c a} \widetilde{E_{c b}}} & =2 R_{b c}^{-1} W_{a c} W_{a b}-R_{a b} R_{a d}^{-1} W_{c d} R_{b e}^{-1} W_{c e} \\
& =R_{b c}^{-1} W_{a c} W_{a b} .
\end{aligned}
$$

When we add a function $\varphi(x, t)$ to $Z$, we can get the following evolution equation $\square(Z+\varphi)=R(Z+\varphi)+2 X_{a} U_{a}+A_{a b} V_{a} V_{b}-R_{a b} \widetilde{Y_{c a}} \widetilde{Y_{c b}}+\left(\square \varphi+R_{b c}^{-1} W_{a c} W_{a b}-R \varphi\right)$.

When $0 \leq R_{a b} \leq M g_{a b}$, we have $R_{b c}^{-1} W_{a c} W_{a b} \geq \frac{1}{M} W_{a b} W_{a b}$. So it is just the good positive term to make $\square \varphi+R_{b c}^{-1} W_{a c} W_{a b}-R \varphi$ positive. We will show the details in Section 5 . 
4. Estimate of another extra term. In fact, the Harnack inequality of Chow holds for the complete locally conformally flat manifolds with nonnegative Ricci curvature in [3]. However, in this paper the Ricci curvature satisfies the condition ( $\star$ ). In order to make the similar argument with Chow [2], we need to add one positive term to the Ricci curvature such that it become nonnegative. So the quantities of Definition 3.1 will need to make a corresponding change and the evolution equation of the basic Harnack expression will have another extra term. We denote it by CNS. In this section we will obtain its lower bound.

Firstly, we change the quantities in Definition 3.1 as follows.

Definition 4.1. We let

$$
\begin{aligned}
& \widetilde{R_{a b}}=R_{a b}+m(t) g_{a b}, \quad \widetilde{R}=R+n m(t), \\
& \widetilde{X_{a}}=\frac{1}{2} \nabla_{a} R+\frac{1}{2(n-1)} \widetilde{R_{a b}} V_{b}, \quad \widetilde{Y_{a b}}=\nabla_{a} V_{b}-R g_{a b}+\widetilde{E_{a b}}, \\
& \widetilde{W_{a b}}=(n-1) \nabla_{a} \nabla_{b} R+\nabla_{a} R_{b c} V_{c}+R \widetilde{R_{a b}}, \\
& \widetilde{Z}=\partial_{t} R+V_{a} \nabla_{a} R+\frac{1}{2(n-1)} \widetilde{R_{a b}} V_{a} V_{b}+n m(t) R, \\
& \widetilde{W}=\partial_{t} R+\frac{1}{2} \nabla_{a} R V_{a}+n m(t) R, \\
& \widetilde{U_{a}}=\square V_{a}-\frac{(n-1)(n-2)}{2} \nabla_{a} R-\frac{n}{2} \widetilde{R_{a b}} V_{b}+\widetilde{R} V_{a} .
\end{aligned}
$$

where $\widetilde{E_{a b}}$ will be defined later.

Recalling the evolution equation (3.1) of $Z$ in Section 3, we hope $\square \widetilde{Z}$ have the following form

$$
\begin{aligned}
\square \widetilde{Z}= & R \widetilde{Z}+2 \widetilde{X_{a}} \widetilde{U_{a}}+\widetilde{A_{a b}} V_{a} V_{b}-\widetilde{R_{a b}} \widetilde{Y_{c a}} \widetilde{Y_{c b}}-2 \widetilde{W_{a b}} \widetilde{Y_{a b}}+2 \widetilde{R_{a b}} \widetilde{E_{c a}} \widetilde{Y_{c b}} \\
& +2 \widetilde{W_{a b}} \widetilde{E_{a b}}-\widetilde{R_{a b}} \widetilde{E_{c a}} \widetilde{E_{c b}}+C N S .
\end{aligned}
$$

where $\widetilde{A_{a b}}=\frac{1}{2(n-1)(n-2)} \widetilde{B_{a b}}+\frac{1}{2(n-1)}\left(n{\widetilde{R_{a b}}}^{2}-\widetilde{R} \widetilde{R_{a b}}\right)$ and $\widetilde{B_{a b}}=(n-1)\left|\widetilde{R_{a b}}\right|^{2} g_{a b}+$ $n \widetilde{R} \widetilde{R_{a b}}-n(n-1){\widetilde{R_{a b}}}^{2}-\widetilde{R}^{2} g_{a b}$.

Lemma 4.1. If the Ricci curvature satisfies $R_{a b} \geq-m g_{a b}$ on $M^{n}$, then

$$
A_{a b} \geq 0 \text {. }
$$

Proof. For $\forall p \in M$, and $\forall V \in T_{p} M$, we know $\widetilde{A_{a b}} V_{a} V_{b} \geq 0$ from [2]. By simple calculating, we have $\left(A_{a b}-\widetilde{A_{a b}}\right) V_{a} V_{b}=0$. Thus the lemma holds. $\square$

So from (3.1)(4.1) and the proof of Lemma 4.1, we get

$$
\begin{aligned}
C N S= & \square(\widetilde{Z}-Z)+R(Z-\widetilde{Z})+2\left(X_{a} U_{a}-\widetilde{X_{a}} \widetilde{U_{a}}\right)+\left(\widetilde{R_{a b}}-R_{a b}\right) \widetilde{Y_{c a}} \widetilde{Y_{c b}} \\
& +2 \widetilde{Y_{a b}}\left(\widetilde{W_{a b}}-W_{a b}\right)+2\left(R_{a b}-\widetilde{R_{a b}}\right) \widetilde{E_{c a}} \widetilde{Y_{c b}}+2 \widetilde{E_{a b}}\left(W_{a b}-\widetilde{W_{a b}}\right) \\
& +\left(\widetilde{R_{a b}}-R_{a b}\right) \widetilde{E_{c a}} \widetilde{E_{c b}} .
\end{aligned}
$$

Proposition 4.1. Suppose $\left(M^{n}, g(x, t)\right)$ satisfies the same conditions as Theorem 1.1, then at $\forall(x, t) \in B_{\frac{r}{2}}(O, t) \times\left[0, r^{2}\right], \forall V \in T_{x} M^{n}$, we have

$$
C N S \geq-C M^{2}\left(\frac{1}{r^{2}}+\frac{1}{t}+M\right),
$$


where $C$ depends only on the dimension $n$.

Proof. From (4.2), we have the following estimate at $(x, t) \in B_{\frac{r}{2}}(O, t) \times\left[0, r^{2}\right]$ for $\forall V \in T_{x} M^{n}$, by using the curvature condition $(\star)$ and Theorem 2.1 .

$$
\begin{aligned}
C N S & =n\left(m^{\prime}(t)-m R\right) R+\frac{|V|^{2}}{2(n-1)}\left(m^{\prime}(t)-3 m R-n m^{2}\right)-m V_{a} \nabla_{a} R \\
& \geq n\left(m^{\prime}(t)-n m M\right) R+\frac{|V|^{2}}{2(n-1)}\left(m^{\prime}(t)-(4 n+1) m M\right)-\frac{n-1}{2}|\nabla R|^{2} \\
& \geq-C M^{2}\left(\frac{1}{r^{2}}+\frac{1}{t}+M\right) . \quad \mathbf{~}
\end{aligned}
$$

5. The local Harnack estimate. In this section we will first give the proof of Theorem 1.1, and then prove Corollary 1.1 by using the local Harnack estimate.

If we add some fucntion $\varphi(x, t)$ to $\widetilde{Z}$, and set $\widetilde{E_{a b}}={\widetilde{R_{b c}}}^{-1} \widetilde{W_{a c}}$, then we get the equality

$$
\begin{aligned}
\square(\widetilde{Z}+\varphi)= & R(\widetilde{Z}+\varphi)+2 \widetilde{X_{a}} \widetilde{U_{a}}+\widetilde{A_{a b}} V_{a} V_{b}-\widetilde{R_{a b}} \widetilde{Y_{c a}} \widetilde{Y_{c b}}+\widetilde{R_{b c}}-1 \widetilde{W_{a b}} \widetilde{W_{a c}} \\
& +C N S+\square \varphi-R \varphi .
\end{aligned}
$$

If $-m(t) g_{a b}(t) \leq R_{a b}(t) \leq M g_{a b}(t)$, then $\widetilde{A_{a b}} \geq 0$ and $0 \leq \widetilde{R_{a b}} \leq(M+m(t)) g_{a b}$, so ${\widetilde{R_{a b}}}^{-1} \geq \frac{1}{M+m(t)} g_{a b}$.

So

$$
\begin{aligned}
\widetilde{R_{b c}}-1 \widetilde{W_{a b} \widetilde{W_{a c}} \geq} \geq & \frac{1}{M+m(t)} \widetilde{W_{a b} \widetilde{W_{a b}}} \\
= & \frac{1}{M+m(t)}\left|\widetilde{W_{a b}}+\frac{\varphi}{n} g_{a b}\right|^{2}-\frac{2}{M+m(t)}(\widetilde{W}+\varphi) \frac{\varphi}{n} \\
& +\frac{1}{M+m(t)} \frac{\varphi^{2}}{n} .
\end{aligned}
$$

Then we have

$$
\begin{aligned}
\square(\widetilde{Z}+\varphi) \geq & R(\widetilde{Z}+\varphi)+2 \widetilde{X_{a}} \widetilde{U_{a}}-\widetilde{R_{a b}} \widetilde{Y_{c a}} \widetilde{Y_{c b}}+\frac{1}{M+m(t)}\left|\widetilde{W_{a b}}+\frac{\varphi}{n} g_{a b}\right|^{2} \\
& -\frac{2}{M+m(t)}(\widetilde{W}+\varphi) \frac{\varphi}{n}+\left(\square \varphi+\frac{1}{M+m(t)} \frac{\varphi^{2}}{n}-R \varphi+C N S\right) .
\end{aligned}
$$

Now we prove Theorem 1.1.

Proof of Theorem 1.1. Let $\varphi=B M\left(M+\frac{r^{2}}{\left(r^{2}-4 d^{2}\right)^{2}}+\frac{1}{t}\right)$, where $B$ will be chosen later. We see $\varphi=+\infty$ at $B_{\frac{r}{2}}(O, 0) \cup \partial B_{\frac{r}{2}}(O, t), t \in\left[0, r^{2}\right]$. If $\widetilde{Z}+\varphi$ first attain zero in $B_{\frac{r}{2}}(O, t) \times\left[0, r^{2}\right]$ at $\left(x_{0}, t_{0}\right)$ for some vector $V$, then it must be $\left(x_{0}, t_{0}\right) \in$ $\operatorname{int}\left(B_{\frac{r}{2}}(O, t)\right) \times\left(0, r^{2}\right]$. And we know

$$
0=\left.\frac{\partial(\widetilde{Z}+\varphi)(V+s \widetilde{V})}{\partial s}\right|_{s=0}=2 \sum_{a} \widetilde{X_{a}} \widetilde{V_{a}}, \text { for } \forall \quad \widetilde{V} \in T_{x_{0}} M^{n} .
$$

So we get $\widetilde{X_{a}}=0$. On the other hand, we have $\widetilde{Z}+\varphi-\widetilde{X_{a}} V_{a}=\widetilde{W}+\varphi=0$. We extend $V$ to one vector field in space-time such that $\widetilde{Y_{a b}}=0$, and then by Proposition 
4.1 at the point we get

$$
\begin{aligned}
\square(\widetilde{Z}+\varphi) & \geq \square \varphi+\frac{1}{M+m(t)} \frac{\varphi^{2}}{n}-R \varphi-C M^{2}\left(\frac{1}{r^{2}}+\frac{1}{t}+M\right) \\
& \geq-\frac{C}{B M} \varphi^{2}-\frac{C}{B M} \varphi^{2}+\frac{1}{2 M} \frac{\varphi^{2}}{n}-\frac{C}{B M} \varphi^{2}-\frac{C}{B^{2} M} \varphi^{2} \\
& \geq-\frac{C}{B M} \varphi^{2}+\frac{1}{2 M} \frac{\varphi^{2}}{n}-\frac{C}{B^{2} M} \varphi^{2} \\
& =\frac{\varphi^{2}}{2 n M B^{2}}\left(B^{2}-2 n B C-2 n C\right) \\
& >0 .
\end{aligned}
$$

The second inequality holds by the standard Laplacian comparison theorem and $d \leq \frac{r}{2} \leq \frac{\theta}{2 \sqrt{M}}$. The last inequality holds when we choose $B>n C+\sqrt{n^{2} C^{2}+2 n C}$. So this is a contradiction. We complete the proof of the theorem. $\square$

Now we can prove the corollary.

Proof of Corollary 1.1. At $(O, t)$, for $\forall V \in T_{O} M^{n}$, by Theorem 1.1 we have

$$
\partial_{t} R+n m(t) R+\nabla R(V)+\frac{1}{2(n-1)}\left(R_{a b}+m(t) g_{a b}\right) V_{a} V_{b}+B M\left(\frac{1}{r^{2}}+\frac{1}{t}+M\right) \geq 0 .
$$

Then if $V=0$, we get $\partial_{t} R+n m(t) R+B M\left(\frac{1}{r^{2}}+\frac{1}{t}+M\right) \geq 0$. Meanwhile for $\forall$ $\lambda \in \mathbb{R}$, we also have

$$
\frac{\lambda^{2}}{2(n-1)}\left(R_{a b}+m(t) g_{a b}\right) V_{a} V_{b}+\lambda \nabla R(V)+\partial_{t} R+n m(t) R+B M\left(\frac{1}{r^{2}}+\frac{1}{t}+M\right) \geq 0 .
$$

Thus we obtain

$$
|\nabla R(V)|^{2} \leq \frac{2}{n-1}\left(R_{a b}+m(t) g_{a b}\right) V_{a} V_{b}\left[\partial_{t} R+n m(t) R+B M\left(\frac{1}{r^{2}}+\frac{1}{t}+M\right)\right] .
$$

By Theorem 2.1 and the curvature condition $(\star)$, at $\left(O, r^{2}\right)$ we know

$$
\begin{aligned}
& \partial_{t} R+n m(t) R+B M\left(\frac{1}{r^{2}}+\frac{1}{t}+M\right) \\
= & \left.(n-1) \Delta R+R^{2}+n m\left(r^{2}\right) R+B M\left(\frac{2}{r^{2}}+M\right)\right) \\
\leq & C M\left(\frac{2}{r^{2}}+M\right)+C M^{2}+B M\left(\frac{2}{r^{2}}+M\right) \\
\leq & C_{1} M^{2}+C_{2} M .
\end{aligned}
$$

So for $M \geq 1$,

$$
|\nabla R(V)|^{2} \leq C_{1} M^{2}\left(R_{a b}+m(t) g_{a b}\right) V_{a} V_{b} ;
$$

and for $0<M<1$,

$$
|\nabla R(V)|^{2} \leq C_{2}\left(R_{a b}+m(t) g_{a b}\right) V_{a} V_{b} .
$$

It is clear for the third case. 
6. Remark. 1. The local Harnack inequality can not be got from gradient estimate directly. Set $V_{a}=-{\widetilde{R_{a b}}}^{-1} \nabla_{b} R$, then

$$
\nabla R(V)+\frac{1}{2(n-1)} \widetilde{R_{a b}} V_{a} V_{b}=-\frac{2 n-3}{2(n-1)}{\widetilde{R_{a b}}}^{-1} \nabla_{a} R \nabla_{b} R,
$$

We know $B M\left(M+\frac{r^{2}}{\left(r^{2}-4 d^{2}\right)^{2}}+\frac{1}{t}\right)$ can control $\partial_{t} R+n m(t) R$ by gradient estimates, but $-\infty \leq-{\widetilde{R_{a b}}}^{-1} \leq-\frac{1}{M+m(t)} g_{a b}$, so the lower bound of $-{\widetilde{R_{a b}}}^{-1} \nabla_{a} R \nabla_{b} R$ can't be found.

2. It is hard to get the local Harnack inequaliy directly without using the good extra term.

3. The additive term $n m(t) R$ is used to make the calculation easier, but it is not the only way, one can also add something else.

Acknowledgement. I am grateful to Professor Kefeng Liu for his help in my study. I would like to thank Jie Wang for his paper and his good advice during this work, and especially thank Professor R. Hamilton for his wonderful report on the fourth ICCM.

\section{REFERENCES}

[1] H. D. CaO And X. P. Zhu, A complete proof of the Poincaré and Geometrization conjecturesApplication of the Hamilton-Perelman theory of the Ricci flow, Asian J. Math., 10 (2006), pp. 165-492.

[2] Ben. Chow, The Yamabe flow on locally conformally flat manifolds with positive Ricci curvature, Comm. Pure Appl. Math., 45 (1992), pp. 1003-1014.

[3] H. L. Gu, Manifolds with pointwise Ricci pinched curvature, arXiv:math.DG/0707.0034 v1 June 30, 2007, preprint.

[4] J. WANG, Local Harnack estimate for mean curvature flow in Euclidean space, preprint. 